\title{
Partonic effects on anisotropic flows at RHIC
}

\author{
Lie-Wen Chen $\dagger \ddagger$ and Che Ming Ko \\ Cyclotron Institute and Physics Department, Texas A\&M University, College \\ Station, Texas 77843-3366
}

\begin{abstract}
We report recent results from a multiphase transport (AMPT) model on the azimuthal anisotropies of particle momentum distributions in heavy ion collisions at the Relativistic Heavy Ion Collider. These include higher-order anisotropic flows and their scaling, the rapidity dependence of anisotropic flows, and the elliptic flow of charm quarks.
\end{abstract}

PACS numbers: 25.75.Ld, 24.10.Lx

\section{Introduction}

Anisotropic flows in heavy ion collisions at the Relativistic Heavy Ion Collider (RHIC) are sensitive to the properties of produced matter. This sensitivity not only exists in the larger elliptic flow [1, 2, 3, 4, 5, 6, 7, but also in the smaller higher-order anisotropic flows [8, 9, 10. Experimentally, scaling relations among hadron anisotropic flows have been observed [11, and according to the quark coalescence model they are related to similar scaling relations among parton anisotropic flows [10. Also, anisotropic flows measured at finite pseudorapidities are seen to depend strongly on the rapidity [11, 12, 13, 14, 15, 16, and this has so far not been reproduced by theoretical models [17, 18, 19, 20]. For charmed mesons, the observed transverse momentum spectra of electrons from their decays are found to be consistent with both limiting scenarios of perturbative QCD spectra without final-state interactions and complete thermalization including transverse expansion [21. The charm elliptic flows given by the quark coalescence model show, however, marked differences between these two scenarios [22]. These interesting phenomena have recently been studied in a multiphase transport (AMPT) model that includes both initial partonic and final hadronic interactions [23, 24, 25, 26, 27, 28. In this talk, we briefly review the AMPT model and discuss the results obtained from this model.

$\dagger$ On leave from Department of Physics, Shanghai Jiao Tong University, Shanghai 200030, China

$\ddagger$ lwchen@comp.tamu.edu 


\section{The AMPT model}

The AMPT model is a hybrid model that uses minijet partons from hard processes and strings from soft processes in the Heavy Ion Jet Interaction Generator (HIJING) model [29] as the initial conditions for modeling heavy ion collisions at ultra-relativistic energies. Time evolution of resulting minijet partons is then described by Zhang's parton cascade (ZPC) [30] model. At present, this model includes only parton-parton elastic scatterings with an in-medium cross section derived from the lowest-order Born diagram with an effective gluon screening mass taken as a parameter for fixing the magnitude and angular distribution of parton scattering cross section. After minijet partons stop interacting, they are combined with their parent strings, as in the HIJING model with jet quenching, to fragment into hadrons using the Lund string fragmentation model as implemented in the PYTHIA program [31]. The final-state hadronic scatterings are then modeled by a relativistic transport (ART) model [32.

Since the initial energy density in $\mathrm{Au}+\mathrm{Au}$ collisions at RHIC is much larger than the critical energy density at which the hadronic matter to quark-gluon plasma transition would occur [27, 33, the AMPT model has been extended by converting the initial excited strings into partons [5]. In this string melting scenario, hadrons, that would have been produced from string fragmentation, are converted instead to valence quarks and/or antiquarks. Interactions among these partons are again described by the ZPC parton cascade model. Since there are no inelastic scatterings, only quarks and antiquarks from the melted strings are present in the partonic matter. The transition from the partonic matter to the hadronic matter is achieved using a simple coalescence model, which combines two nearest quark and antiquark into mesons and three nearest quarks or antiquarks into baryons or anti-baryons that are close to the invariant mass of these partons. The present coalescence model is thus somewhat different from the ones recently used extensively [34, 35, 36, 37] for studying hadron production at intermediate transverse momenta.

\section{Anisotropic flows at midrapidity}

Using the AMPT model with string melting, we have studied anisotropic flows in the pseudorapidity range $|\eta|<1.2$ in minimum bias $\mathrm{Au}+\mathrm{Au}$ collisions at $\sqrt{s}=200 \mathrm{AGeV}$. In Fig. 1, we show the final anisotropic flows $v_{n}$ of charged hadrons, defined by the average $\langle\cos (n \phi)>$ of the azimuthal distributions of their transverse momenta, as functions of transverse momentum $p_{T}$ for parton scattering cross sections $\sigma_{p}=3$ and $10 \mathrm{mb}$, together with recent experimental data from the STAR collaboration [11]. It is seen that the parton scattering cross section $\sigma_{p}=3 \mathrm{mb}$ underestimates the data at higher $p_{T}(>1 \mathrm{GeV} / c)$ while $\sigma_{p}=10 \mathrm{mb}$ seems to give a better fit to the data. The values of hadronic $v_{6}$ are in agreement with the data within error bars, although they are essentially zero. The $v_{4}$ of charged hadrons exhibits a stronger sensitivity to the parton cross section than their $v_{2}$, especially at higher $p_{T}$, and is thus a more sensitive 


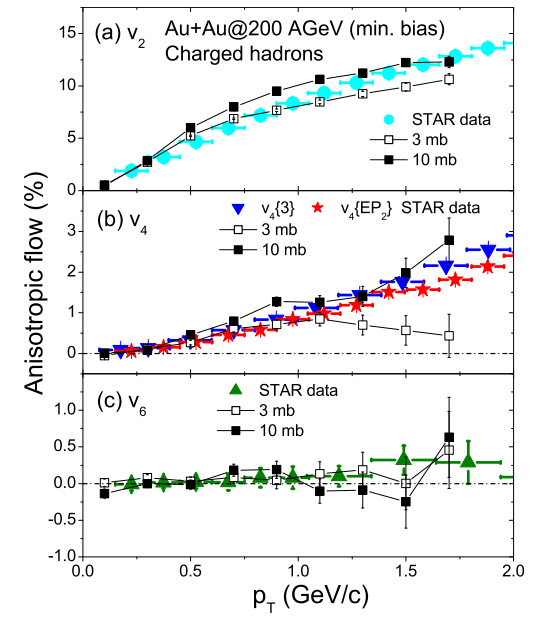

Figure 1: Anisotropic flows $v_{2}(\mathrm{a}), v_{4}(\mathrm{~b})$, and $v_{6}(\mathrm{c})$ of charged hadrons in $|\eta|<1.2$ from minimum bias $\mathrm{Au}+\mathrm{Au}$ collisions at $\sqrt{s}=200 \mathrm{AGeV}$ as functions of $p_{T}$ for parton cross sections of 3 (open squares) and 10 (solid squares) mb. Data are from the STAR Collaboration [1].

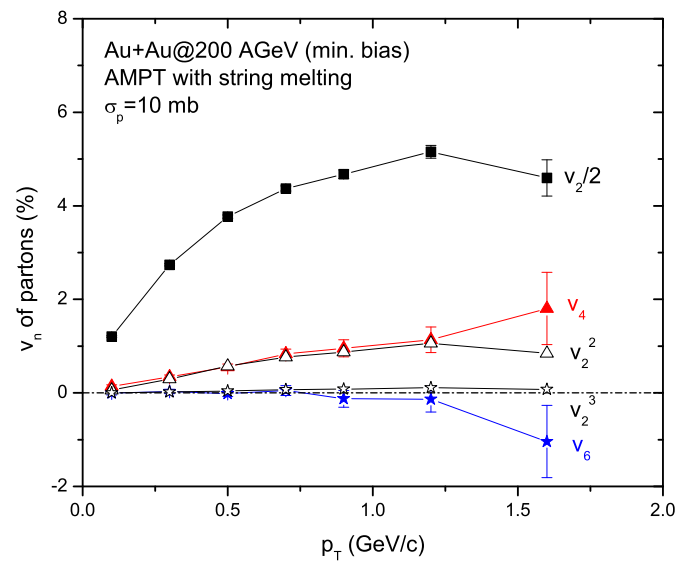

Figure 2: Transverse momentum dependence of midrapidity parton anisotropic flows $v_{2}, v_{4}$ and $v_{6}$ from minimum bias events for $\mathrm{Au}+\mathrm{Au}$ at $\sqrt{s}=200 \mathrm{AGeV}$ with parton scattering cross section $10 \mathrm{mb}$. Also plotted are $v_{2}^{2}$ (open triangles) and $v_{2}^{3}$ (open stars).

probe to the initial partonic dynamics in relativistic heavy ion collisions.

The experimental data shown in Fig. 1 on higher-order anisotropic flows show the scaling relation $v_{n}\left(p_{T}\right) \sim v_{2}^{n / 2}\left(p_{T}\right)$ [1]. In the naive quark coalescence model [37] that only allows quarks with equal momentum to form a hadron, meson higher-order anisotropic flows $v_{4, M}$ and $v_{6, M}$ are related to parton anisotropic flows $v_{n, q}$ by [2, 9, 10,

$$
\frac{v_{4, M}\left(p_{T}\right)}{v_{2, M}^{2}\left(p_{T}\right)} \approx \frac{1}{4}+\frac{1}{2} \frac{v_{4, q}\left(p_{T} / 2\right)}{v_{2, q}^{2}\left(p_{T} / 2\right)}, \quad \frac{v_{6, M}\left(p_{T}\right)}{v_{2, M}^{3}\left(p_{T}\right)} \approx \frac{1}{4}\left(\frac{v_{4, q}\left(p_{T} / 2\right)}{v_{2, q}^{2}\left(p_{T} / 2\right)}+\frac{v_{6, q}\left(p_{T} / 2\right)}{v_{2, q}^{3}\left(p_{T} / 2\right)}\right) .
$$

The meson higher-order anisotropic flows thus satisfy scaling relations if such relations exist among quark higher-order anisotropic flows.

In Fig. 2] we show the $p_{T}$ dependence of the anisotropic flows $v_{2}, v_{4}$ and $v_{6}$ of midrapidity partons obtained from the AMPT model with string melting and a parton scattering cross section of $10 \mathrm{mb}$ for above reaction. Also shown in Fig. 2 are $v_{2}^{2}$ (open triangles) and $v_{2}^{3}$ (open stars). Comparing them to $v_{4}$ and $v_{6}$, respectively, indeed show that the parton anisotropic flows satisfy the scaling relation $v_{n, q}\left(p_{T}\right) \sim v_{2, q}^{n / 2}\left(p_{T}\right)$. With a parton scaling factor of 1 , the naive coalescence model would lead to the following scaling relations for meson anisotropic flows:

$$
\frac{v_{4, M}\left(p_{T}\right)}{v_{2, M}^{2}\left(p_{T}\right)} \approx \frac{3}{4}, \quad \frac{v_{6, M}\left(p_{T}\right)}{v_{2, M}^{3}\left(p_{T}\right)} \approx \frac{1}{2}
$$

The resulting hadron scaling factors of $3 / 4$ and $1 / 2$ are, however, smaller than those $(\sim 1.2)$ extracted from measured anisotropic flows of charged hadrons. Since the naive quark coalescence model does not allow hadron formation from quarks with different 
momenta as in more realistic quark coalescence models [34, 35, 36, it is not expected to give a quantitative description of the experimental observation. Such effects are, nevertheless, included in the AMPT model, which have been shown in Fig. 1 to reproduce measured hadron anisotropic flows.

\section{Pseudorapidity dependence of anisotropic flows}

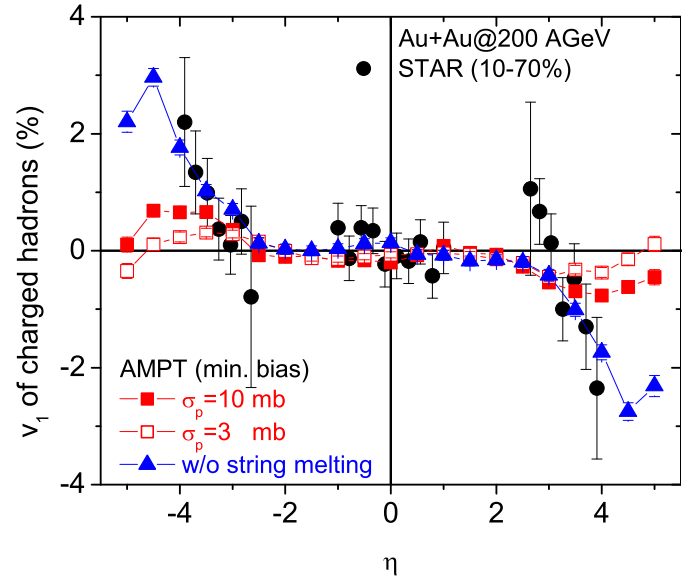

Figure 3: Pseudorapidity dependence of $v_{1}$ from minimum bias events of $\mathrm{Au}$ + Au collisions at $\sqrt{s}=200 \mathrm{AGeV}$ in the string melting scenario with parton scattering cross sections $\sigma_{p}=3$ (open squares) and 10 (solid squares) mb as well as the scenario without string melting (solid triangles). Data are from the STAR collaboration [1].

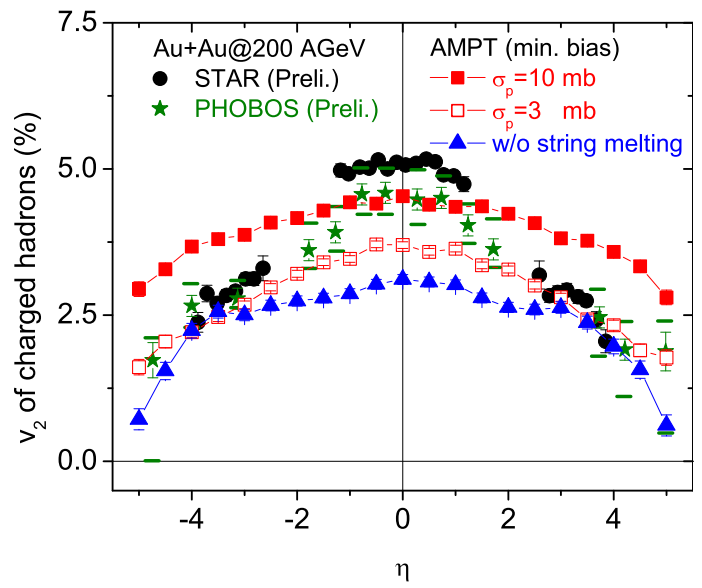

Figure 4: Pseudorapidity dependence of $v_{2}$ from minimum bias $\mathrm{Au}+\mathrm{Au}$ collisions at $\sqrt{s}=200 \mathrm{AGeV}$ in the string melting scenario with parton cross sections of 3 (open squares) and 10 (solid squares) $\mathrm{mb}$ and the scenario without string melting (solid triangles). Data are from the PHOBOS (stars) 13] and STAR collaborations (solid circles) [15].

Results from the AMPT model on the pseudorapidity dependence of $v_{1}$ for charged hadrons from minimum bias events of $\mathrm{Au}+\mathrm{Au}$ collisions at $\sqrt{s}=200 \mathrm{AGeV}$ are shown in Fig. 3 for the scenarios of string melting with parton scattering cross sections $\sigma_{p}=3$ (open squares) and $10 \mathrm{mb}$ (solid squares) as well as for the scenario without string melting (solid triangles). Also included in Fig. 3 are recent data from the STAR collaboration (solid circles) 11. Both scenarios can reproduce approximately the data around the mid-pseudorapidity region, i.e., $v_{1}$ is flat (essentially zero) around mid- $\eta$. For $v_{1}$ at large $|\eta|$, the string melting scenario with both parton scattering cross sections $\sigma_{p}=3 \mathrm{mb}$ and $10 \mathrm{mb}$ underestimates significantly the data. On the other hand, the scenario without string melting seems to give a good description of $v_{1}$ at large $|\eta|$.

The predicted pseudorapidity dependence of charged hadron $v_{2}$ from the same reaction is shown in Fig. 4. together with preliminary data from the PHOBOS collaboration (solid stars) [13] and the STAR collaboration (solid circles) [15]. The 
string melting scenario with $\sigma_{p}=10 \mathrm{mb}$ (solid squared) is seen to describe very well the data for $v_{2}$ around mid- $\eta(|\eta| \leq 1.5)$ but overestimates the data at large pseudorapidity. The overestimation of may stem from the use of constant parton scattering cross section in the AMPT model. Since the properties of partonic matter at different rapidities may not be the same in heavy ion collisions at RHIC, different parton cross sections may have to be used. Comparison between theoretical results and the experimental data for elliptic flow indicates that a larger $\sigma_{p}=10 \mathrm{mb}$ is needed at midrapidity but a smaller $\sigma_{p}=3 \mathrm{mb}$ (open squares) gives a better description at large pseudorapidity. Also shown in Fig. 4 are results obtained from the scenario without string melting (solid triangles), and they are seen to also describe the data at large pseudorapidity $(|\eta| \geq 3)$. Therefore, the scenario without string melting can describe simultaneously the data on $v_{1}$ and $v_{2}$ at large pseudorapidity $(|\eta| \geq 3)$. These interesting features imply that initially the matter produced at large pseudorapidity $(|\eta| \geq 3)$ is dominated by strings while that produced around mid-rapidity $(|\eta| \leq 3)$ mainly consists of partons. This is a reasonable picture as particles at large rapidity are produced later in time when the volume of the system is large and the energy density is small.

\section{Charm flows at RHIC}

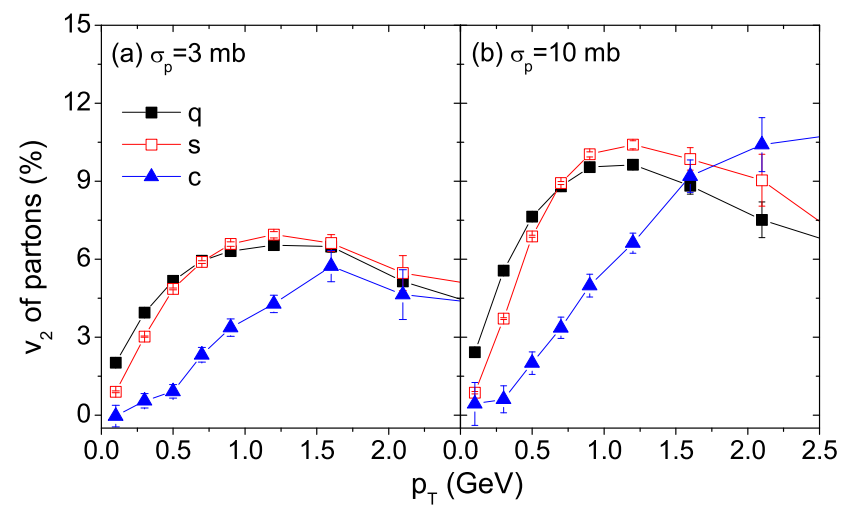

Figure 5: Transverse momentum dependence of $v_{2}$ for different parton flavors in midrapidity from minimum bias $\mathrm{Au}+\mathrm{Au}$ collisions at $\sqrt{s}=200 \mathrm{AGeV}$. Results are for parton cross sections $\sigma_{p}=3$ (a) and $10 \mathrm{mb}(\mathrm{b})$.

Using the AMPT model in the string melting scenario with same parton scattering cross section of $\sigma_{p}=3$ or $10 \mathrm{mb}$ for all quarks, we have also studied the $p_{T}$-dependence of $v_{2}$ for partons of different flavors in the midrapidity from minimum bias $\mathrm{Au}+\mathrm{Au}$ collisions at $\sqrt{s}=200 \mathrm{AGeV}$. Using the current quark mass of $10 \mathrm{MeV}$ for $d$ quark, 6 $\mathrm{MeV}$ for $u$ quark, $200 \mathrm{MeV}$ for $s$ quark, and $1.35 \mathrm{GeV}$ for $c$ quark, the results are shown in Fig. 5. It is seen that the quark $v_{2}$ increases with increasing $p_{T}$, and at $p_{T}<1.5$ $\mathrm{GeV} / \mathrm{c}$ it is smaller for the heavier $c$ quark than for the lighter $d$ and $s$ quarks. This mass 
dependence of $v_{2}$ at low $p_{T}$ is similar to the mass ordering of hadron elliptic flows in the hydrodynamic model which assumes that the matter is in local thermal equilibrium and thus develops a large collective radial flow. However, instead of continuing increase of $v_{2}$ with respect to $p_{T}$ as in the hydrodynamic model, the parton $v_{2}$ in the transport model reaches a maximum value at certain large $p_{T}$, indicating that high momentum particles do not achieve thermal equilibrium with the bulk matter. These features are seen for both values of parton scattering cross sections, except that the value of $v_{2}$ is larger for a larger parton scattering cross section. As in the case of light hadrons, study of charmed hadron flows offers the possibility to understand the charm quark interactions in the quark-gluon plasma.

\section{Summary and discussions}

In summary, using the AMPT model, we have studied the partonic effects on anisotropic flows in heavy ion collisions at RHIC. We find that measured $v_{2}, v_{4}$ and $v_{6}$ of charged hadrons at midrapidity in $\mathrm{Au}+\mathrm{Au}$ collisions at $\sqrt{s}=200 \mathrm{AGeV}$ can be described by a parton scattering cross section of about $10 \mathrm{mb}$ and that $v_{4}$ is a more sensitive probe to the initial partonic dynamics in these collisions than $v_{2}$. Moreover, higher-order parton anisotropic flows are nonnegligible and satisfy the scaling relation $v_{n, q}\left(p_{T}\right) \sim v_{2, q}^{n / 2}\left(p_{T}\right)$, which leads naturally to the observed similar scaling relation among hadron anisotropic flows if the partonic matter hadronizes via the coalescence of quarks and antiquarks. The results on the rapidity dependence of anisotropic flows suggest that a partonic matter is formed during early stage of relativistic heavy ion collisions only around midrapidity and that strings remain dominant at large rapidities. We have also studied the elliptic flow of charm quarks by using different scattering cross sections, and find that it has a maximum value similar to that for light quarks and is also sensitive to the charm quark scattering cross sections. The charm quark elliptic flow shows, on the other hand, a very different dependence on the transverse momentum due to its large mass.

In reproducing the experimental data on hadron anisotropic flows, a large parton cross section of about $10 \mathrm{mb}$ is needed in the AMPT model. Comparing to the cross section given by the perturbative QCD, this value is an order of magnitude larger, indicating that nonperterbative effects are important in the quark-gluon plasma. Indeed, recent lattice gauge studies have shown that hadron spectral functions including those consisting of heavy charm quarks survive in the quark-gluon plasma [38, 39]. Including the effect of these quasi bound states is expected to enhance the parton-parton scattering cross section.

\section{Acknowledgment}

We thank V. Greco, P. F. Kolb, Z. W. Lin, and B. Zhang for collaboration on different parts of the presented work. This paper was based on work supported by the US National Science Foundation under Grant No. PHY-0098805 and the Welch Foundation under 
Grant No. A-1358. LWC was also supported by the National Science Foundation of China under Grant No. 10105008.

\section{References}

[1] D. Teaney, J. Laureant, and E.V. Shuryak, Phys. Rev. Lett. 86, 4783 (2001).

[2] P. F. Kolb, P. Huovinen, U. Heinz, and H. Heiselberg, Phys. Lett. B 500, 232 (2001).

[3] B. Zhang, M. Gyulassy, and C. M. Ko, Phys. Lett. B455, 45 (1999)

[4] D. Molnar and M. Gyulassy, Nucl. Phys. A698, 379 (2002).

[5] Z. W. Lin and C. M. Ko, Phys. Rev. C 65, 034904 (2002).

[6] M. Gyulassy, I. Vitev and X. N. Wang, Phys. Rev. Lett. 86, 2537 (2001).

[7] S. A. Voloshin, Nucl. Phys. A715, 379c (2003).

[8] P. F. Kolb, Phys. Rev. C 68, 031902 (R) (2003).

[9] L. W. Chen, C. M. Ko, and Z. W. Lin, Phys. Rev. C 69, 031901 (R) (2004).

[10] P. Kolb, L. W. Chen, V. Greco, and C. M. Ko, Rev. C 69, 051901 (R) (2004).

[11] J. Adams et al. [STAR Collaboration], Phys. Rev. Lett. 92, 062301 (2004).

[12] B.B. Back et al., Phys. Rev. Lett. 89, 222301 (2002).

[13] S. Manly for the PHOBOS Collaboration, Nucl. Phys. A715, 611c (2003).

[14] M. B. Tonjes for the PHOBOS Collaboration, nucl-ex/0403025.

[15] M. D. Oldenberg for the STAR Collaboration, 2004, nucl-ex/0403007.

[16] B.B. Back et al., nucl-ex/0407012.

[17] T. Hirano, Phys. Rev. C 65, 011901 (R) (2001).

[18] U. Heinz and P. F. Kolb, nucl-th/0403044.

[19] M. Csanad, T. Csorgo, B. Lorstad, and A. Ster, nucl-th/0403074.

[20] L.W. Chen, V. Greco, C.M. Ko, and P. F. Kolb, nucl-th/0408021

[21] S. Batsouli, S. Kelly, M. Gyulassy, and J.L. Nagle, Phys. Lett. B557, 26 (2003).

[22] V. Greco, C.M. Ko, and R. Rapp, Phys. Lett. B595, 202 (2004).

[23] B. Zhang, C. M. Ko, B. A. Li, and Z. W. Lin, Phys. Rev. C 61, 067901 (2000).

[24] Z. W. Lin, S. Pal, C. M. Ko, B. A. Li, and B. Zhang, Phys. Rev. C 64, 011902 (2001); Nucl. Phys. A698, 375 (2002).

[25] Z. W. Lin, C. M. Ko, and S. Pal, Phys. Rev. Lett. 89, 152301 (2002).

[26] C.M. Ko, Z.W. Lin, and S. Pal, Heavy Ion Phys. 17, 219 (2003).

[27] B. Zhang, C. M. Ko, B. A. Li, Z. W. Lin, and B. H. Sa, Phys. Rev. C 62, 054905 (2000); B. Zhang, C. M. Ko, B. A. Li, Z. W. Lin, and S. Pal, ibid. 65, 054909 (2002).

[28] S. Pal. C. M. Ko, and Z. W. Lin, Nucl. Phys. A730, 143 (2004).

[29] X. N. Wang and M. Gyulassy, Phys. Rev. D 44, 3501 (1991).

[30] B. Zhang, Comput. Phys. Commun. 109, 193 (1998).

[31] T. Sjostrand, Comput. Phys. Commun. 82, 74 (1994).

[32] B. A. Li and C. M. Ko, Phys. Rev. C 52, 2037 (1995); B.A. Li, A.T. Sustich, B. Zhang, and C.M. Ko, Int. Jour. Phys. E 10, 267-352 (2001).

[33] D. Kharzeev and M. Nardi, Phys. Lett. B507, 121 (2001).

[34] V. Greco, C.M. Ko, and P. Lévai, Phys. Rev. Lett. 90, 202302 (2003); Phys. Rev. C 68, 034904 (2003).

[35] R.C. Hwa and C.B. Yang, Phys. Rev. C 67, 034902 (2003); 064902 (2003).

[36] R.J. Fries, B. Müller, C. Nonaka, and S.A. Bass, Phys. Rev. Lett. 90, 202303 (2003); Phys.Rev. C 68, 044902 (2003).

[37] D. Molnar and S. A. Voloshin, Phys. Rev. Lett. 91, 092301 (2003).

[38] S. Datta, F. Karsch, P. Petreczky, and I. Wetzorke, Phys. Rev. D 69, 0945407 (2004).

[39] M. Asakawa, T. Hatsuda, and Y. Nakahara, Nucl. Phys. A715, 863 (2003); M. Asakawa and T. Hatsuda, Phys. Rev. Lett. 92, 012001 (2003). 